RELATIVISTIC ELECTRON COOLING AND INTRABEAM SCATTERING*

A. G. Ruggiero

November 1984

*Invited talk to the Conference on Electron Cooling, Karlsruhe, West Germany, Sept. 24-26, 1984. 


\title{
RELATIVISTIC ELECTRON COOLING AND INTRABEAM SCATTERING *
}

\author{
A.G. Ruggiero \\ Fermilab, P.O. Box 500, Batavia, IL 60510
}

Introduction

In this paper we discuss two issues one of which concerning the usefulness of relativistic electron cooling for large hadron colliders, and the other the limit on the performance of this from intrabeam scattering. The background we have chosen for our analysis is made speciflcally for proton-antiproton colliders, not just as workable examples but primarily, as we shall see later, for reasons of need, though our results apply to a proton-proton collider as well.

By relativistic electron cooling we do not mean only the instance when $\beta-1$ for both beams but also the case where the electron beam is made of bunches circulating in a storage ring. In this configuration originally proposed by C. Rubbia ${ }^{1}$ very high electron current densities can be reached. In FIg. 1 we show a possible proton-antiproton collider with two electron storage rings, one for cooling of the protons and the other for cooling of the antiprotons. It is well known that electron cooling is independent of the sign of the particle charge, but the two beams have to move in the same direction of the electron bunches to which they have also to match in velocity. We consider three examples; the collider in CERN (SpDSS), at Fermilab (Tev I) and a possible Superconducting Super Collider ( $\vec{p}$ SSC). The first is already operating, ${ }^{2}$ the second is in construction ${ }^{3}$ and the third just a possibility." In Table I we give the comparison of the performance for these three colliders. We can make the following remarks: (i) the emittance values assumed or measured are about the same; ( $i i)$ the beam-beam tune-shift per crossing in the Spps turned out to be larger ${ }^{5}$ then what it was thought to be allowed for beam stability; a value of $\Delta v=0.005$ seems to be now reachable, though what really seems to count is the total tune spread in each beam, rather than the shift itself; (iii) the number of antiprotons required is too large; there is definitively the need to find a method to reduce the filling time without reducing the performance; (iv) intrabeam scattering was found in the Spps to be a serious limitation to the luminosity lifetime ${ }^{6}$ and it is expected to play an important role also in the other two colliders. In Figs. 2 and 3 which have been taken from ref. 6 , we show the effect of intrabeam scattering in the Spp̄s collider.

\section{Performance of Proton-Antiproton Colliders}

The performance of the collider is measured by the luminosity $L$ and the beam-beam tune-shift per crossing $\Delta \nu_{B B}$. For crossing at very small angle $\alpha$ (total) 


$$
-L=\frac{p_{p}^{N-B f} r e v}{4 \pi \sigma^{2} f}
$$

and

$$
\Delta \nu_{B B}=\frac{3 N_{p} r_{p}}{\pi \varepsilon_{N}(1+f)}
$$

where $N_{p}$ and $N_{-}^{-}$are the number of protons and antiprotons per bunch, $B$ the total number $P$ of Bunches per beam, $f_{r e v}$ the revolution frequency, $\sigma$ the rms cross-section of the beam at the collision point, $\varepsilon_{N}$ the normalized beam emittance for $95 \%$ of the population, $r_{p}=1.535 \times 10^{-18} \mathrm{~m}$ and

$$
f=\sqrt{1+p^{2}} \quad \text { with } \quad p=\alpha \sigma e^{12 \sigma}
$$

and $\sigma_{e}$ the rms bunch length.

Let us make the reduction of the number of antiprotons to be our goal. It is then seen from eq. (1) the $\mathrm{N}_{\overrightarrow{\mathrm{p}}}$ can be lowered if also $\sigma^{2}$, that is the emittance $\varepsilon_{\mathrm{N}}$, is also reduced. For obvious reasons it is important to reduce the emittance of both beams since it is usually assumed the two beams have the same emittance to start with. Inspection of eq. (2) then shows one has also to lower the number of protons per bunch, if we require to maintain the value of $\Delta v_{B B}$ within an acceptable limit. For instance if it is possible to find a method to reduce the emittance by a factor of four, the product $\mathrm{N}_{\mathrm{p}} \mathrm{N}_{\mathrm{p}}$ can also be reduced by the same factor; we can allow both $\mathrm{N}_{\mathrm{p}}$ and $\mathrm{N}_{\overline{\mathrm{p}}}$ to change by a common factor which in this case is two. It turns out that the tune-shift $\Delta v_{B B}$ and the betatron phase space density $N / \varepsilon_{N}$ (a parameter which is important to estimate the intrabeam scattering) are both increased also by a factor of two. An example is worked out in Table I following the same criteria for the three colliders. In this table we give the beam-beam tune-shift, versus the emittance, as well as the number of particles per bunch $N$ and the density $N / \varepsilon_{N}$ which we both take equal to one unit for the reference case of $\varepsilon_{N}=24 \pi \mathrm{mm}-\mathrm{mrad}$. As the emittance decreases the beam-beam tune-shift increases beyond what it is believed to be an acceptable value. Clearly the design of a proton-antiproton collider is limited by the allowable beam-beam tune-shift and as a consequence it is not possible to reduce the total number of antiproton required for a given luminosity figure.

\section{The Need for Relativistic Electron Cooling}

It has been found that in the electron-positron storage rings one can allow a much larger beam-beam tune-shift, typically an order of magnitude larger than the one accepted as a limit for hadron colliders. It is believed that the main reason for this is that the electrons betatron and synchrotron trajectories are strongly damped by the synchrotron radiation effects, a mechanism which is quite 
negligible for hadrons at least for energies up to $20 \mathrm{TeV}$. If it is possible to find a damping mechanism also for the protons (antiprotons), fast enough like the synchrotron radiation effects for the electron beams, it should be possible to raise the beam-beam tune-shift by on order of magnitude also for these beams. As one can see from Table II, this could allow 10 times less beam intensity in the $S p \vec{p} S$, or 20 times less in the TeV I and 50 times less in the $p \bar{p}-S S C$, with an equivalent shortening of the antiproton production period and unchanged luminosity performance. It has often been suggested that relativistic electron cooling is the method to create a damping mechanism for protons and antiprotons. We want to investigate this method here again, but use different emphasize as by now it will be obvious to the reader. We will not just be content to preserve the initial beam dimensions with electron cooling against diffusion processes (gas scattering, power supply ripples, $f$ noise...) to lengthen the beam lifetime, but we will look to the possibility of employing electron cooling first to reduce the beam dimensions, and second to provide a fast damping effect with the purpose of minimizing the number of antiprotons.

\section{Intrabeam Scattering}

As we have already pointed out, the betatron phase space density will increase and we expect a limit to the effectiveness of the electron cooling caused by intrabeam scattering. This phenomena has been investigated theoretically in a couple of paper ${ }^{8}, 9$ and it is rather well understood. Several computer codes have been written to estimate diffusion coefficients for a particular beam circulating in a storage ring with assigned lattice. For the Tevatron I project we have made use of a computer code we have obtained from CERN. ${ }^{\circ}$ For the case the beam energy is well above the ring transition energy it is usually found that the energy and radial betatron oscillations are antidamped whereas the vertical motion is damped though at rather slow rate. We take into account the fact that non-linearities in the storage ring, like the beam-beam interaction itself, cause non-linear coupling between the two transverse modes of oscillations difficult to cure; as a consequence the beam will preserve at anytime its "roundness" also in presence of intrabeam scattering, therefore we will denote with $\varepsilon$ the common value of the emittances in the two planes. From the computer code, using the lattice proper for Tevatron $I$, we have derived the following approximate, empirical formula for the diffusion rates due to intrabeam scattering

$$
D_{\beta}=\frac{d \varepsilon}{d t}=\frac{A_{X} I_{p}}{\delta \varepsilon \gamma^{3}}
$$

and

$$
D_{p}=\frac{d \delta}{d t}=\frac{A_{p} I_{p}}{\delta \varepsilon \gamma^{2}}
$$

where $\varepsilon\left(=\sigma^{2} / \beta\right)$ is the actual rms emittance and $\delta$ the rms momentum spread $(\delta p / p)$. 


$$
I_{p}=\frac{N_{p} e \beta c}{\sqrt{2 \pi \sigma_{e}}}
$$

is the peak bunch current. If time is given in hours, the emittance in meter-radians and the current in Ampere then

$$
\begin{aligned}
& A_{x}=0.13 \times 10^{-13} \mathrm{~m}^{2} /(\text { hour } x \text { Amp) } \\
& A_{p}=0.49 \times 10^{-12} \mathrm{~m} /(\text { hour } x \text { Amp) }
\end{aligned}
$$

These values and parametric dependence with $\delta, \varepsilon$ and $\gamma$ are about correct for beam parameters which corresponds to Tevatron I and, likely, also to the Spps. They may not be a representation of the intrabeam scattering effects in the $\mathrm{p} \overline{\mathrm{p}}-\mathrm{SSC}$.

\section{The Theory of Relativistic Electron Cooling}

The theory of relativistic electron cooling has already been established. ${ }^{1}$ It consists mainly of the so-called thermodinamical equations which give the time evolution of the dimensions of both beams (electrons and protons or antiprotons) as they interact with each other. For simplicity in this note we shall make the following approximations: (i) The dimensions of the electron beam are determined only by the synchrotron radiation effects, that is the quantum fluctuation and the radiation damping are of considerably large contribution than the effects from interacting with the hadron beam. Also intrabeam scattering in the electron beam will be ignored compared to the quantum fluctuation. (1i) on the other hand intrabeam scattering will determine the proton beam dimensions more than any other cause, as it is given in equilibrium with coooling effects when interacting with the electron beam.

The following equations then apply to the electron beam

$$
\begin{aligned}
& \frac{d \varepsilon_{e}}{d t}=Q_{\beta}-\frac{2}{\tau} \varepsilon_{e} \\
& \frac{d \delta_{e}}{d t}=Q_{p}-\frac{2}{\tau} \delta_{e}
\end{aligned}
$$

where $Q_{B}$ and $Q_{p}$ are the diffusion coefficents due to quantum fluctuations and $T$ is the synchrotron radiation damping time which we assumed here to be the same for both the rms emittance $\varepsilon_{e}$ and the $r m s$ momentum spread $\delta_{e}$. Eqs. ( $7 a$ and $7 \mathrm{~b}$ ) admits the following equilibrium values

$$
\varepsilon_{e}=\frac{1}{2} \tau Q_{B}
$$




$$
-\bar{\delta}_{e}=\frac{1}{2} \tau Q_{p}
$$

The following equations apply to the proton or antiproton beam ${ }^{1}$

$$
\begin{aligned}
& \frac{d \varepsilon_{p}}{d t}=D_{\beta}-\frac{k_{p} \varepsilon_{p} / \varepsilon_{e}}{\left(\frac{\varepsilon_{p}}{\beta_{p}^{*}}+\frac{\varepsilon_{e}}{\beta_{e}^{*}}\right)^{3 / 2}} \\
& \frac{d \delta_{p}}{d t}=D_{p}-\frac{k_{p} \beta^{2} \gamma^{2} \delta_{p} / 2 \varepsilon_{e}}{\left(\frac{\varepsilon_{p}}{B_{p}^{*}}+\frac{\varepsilon_{e}}{\beta_{e}^{*}}\right)^{3 / 2}}
\end{aligned}
$$

where $D_{B}$ and $D_{p}$ are given by eqs. ( 4 and 5 ), and

$$
\begin{gathered}
k_{p}=\frac{8 r_{e^{r}} I_{e} L_{c} \eta_{p}}{2^{3 / 2} e \beta^{4} \gamma^{5} \beta_{e}} \\
r_{e}=2.818 \times 10^{-25} \mathrm{~m} \\
r_{p}=1.535 \times 10^{-18} \mathrm{~m}
\end{gathered}
$$

I is the peak current of the electron bunch defined by an equation similar to (8). It is obviously required that the two beams have bunches with the same rms length, $\sigma$. $L_{0}-15$ is the Coulomb logarithm and $n_{p}$ is the ratio of the length over which the two beams interact and the circumference of the hadron storage ring. Finally $\beta_{p^{*}}$ and $\beta_{e^{*}}$ are the values of the amplitude lattice functions, in the two rings in the section where the two beams travel together, they are assumed to be the same in both planes. An approximation has been at the demoninator of the r.h. side of both eqs. ( $9 a$ and $b$ ) where we have neglected a term $\left(\delta_{e}{ }^{2}+\delta_{p}{ }^{2}\right) / \gamma^{2}$ compared to the emittance contribution $\left(\varepsilon_{p} / \beta_{p}{ }^{*}+\varepsilon_{e} / \beta_{e} e^{*}\right)$.

Search for the Equilibrium

An equilibrium is found by letting the r.h. side of eqs. ( $9 a$ and $b$ ) to vanish indentically and by taking into account the equilibrium values ( $8 a$ and $b$ ) for the electron beam. We have 


$$
\begin{aligned}
& \frac{A_{x} I_{p}}{\delta_{p} \varepsilon_{p} \gamma^{3}}=\frac{k_{p} \varepsilon / \bar{\varepsilon}_{e}}{\left(\frac{\varepsilon_{\bar{e}}}{\beta_{p}^{*}}+\frac{\bar{E}_{e}}{\beta_{p}^{*}}\right)^{3 / 2}}
\end{aligned}
$$

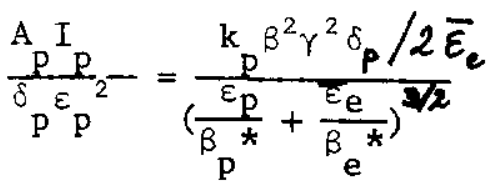

By taking the ratio side by side of these equations we obtain

$$
\delta_{p}=\frac{2 \varepsilon_{p}}{B^{2} \gamma} \frac{A_{p}}{A_{x}}
$$

For the particular application we have chosen this would yield a very small value, in proximity of $10^{-8}$, which we do not believe can be sustained for instance against microwave instability. Therefore we shall assume that the equilibrium value of $\delta$ is the result of microwave stability rather than intrabeam scattering and electron cooling. As a consequence we shall ignore the second equation (11b) and solve the first (11a) for $\varepsilon_{p}$.

Indeed it is well known from experimental observations on electron storage rings that the microwave stability criterion holds despite the presence of synchrotron radiation effects; therefore this should be even more true for an hadron beam is presence of electron cooling.

In Table III we give general parameters for the electron storage ring for each of the case considered. We have chosen strong focussing lattice to reduce the equilibrium emittance. Moreover we have assumed full coupling between the two modes of oscillations so that the electron beam is also "round" to match the "roundness" of the hadron beam. We have taken a bending field of $10 \mathrm{Kgauss}$ and the circumference fraction for cooling $n_{p}=0.0015$. The expected momentum spread $\delta_{p}$ for the hadron team are given in Table $I$.

Results and Discussions

From eq. (11a) we obtain

$$
\varepsilon_{p}^{2}=0.0021 \frac{\beta^{4} \gamma^{2}}{\delta_{p}}\left(\frac{I_{p}}{I_{e}}\right) \beta_{e^{*}} \varepsilon_{e}\left(\frac{\bar{\varepsilon}_{e}}{\beta_{e}^{*}}+\frac{\varepsilon_{p}}{\beta_{p}^{*}}\right)^{3 / 2}
$$

For an efficient cooling it is required that $\bar{\varepsilon}_{e^{\beta}} e^{*}>\varepsilon_{p} p_{p}^{*}$. Let us define

$$
\mathrm{f}_{0}=\frac{\bar{\varepsilon}_{e^{\beta} e^{*}}}{\varepsilon_{p^{\beta} p^{*}}}>1
$$


then

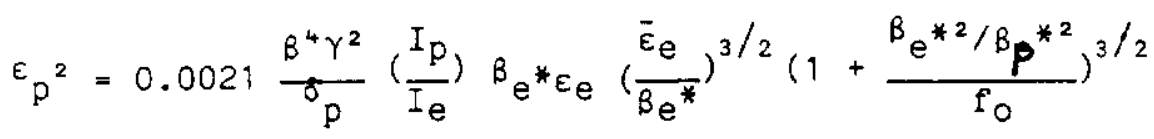

The results are shown in Table IV for $\beta_{*}^{*}=\beta_{e^{*}}$ and $I_{e}=100 I_{p}$ which we believe to be realistic. We immediately see that relation ( 14 ) cannot be satisified by far for the $\bar{p} \bar{p}-S S C$ case, whereas it is satisfied for both the Sp $\bar{p} S$ and Tevatron I. Relativistic electron cooling does not work well for very relatistic energies. For the same reason the technique is quite more effective for the Spps case than for Tev I. The beam intensities shown in Table IV correspond to the calculated equilibrium nomalized emittance $\varepsilon_{\mathrm{N}}$, also shown in this table, and what is required according to Table II to maintain a luminosity performance as described in Table I. Similarly we show also the beam-beam tune-shift which corresponds to the new configuration. The damping time $\tau_{\beta}$ and $\tau_{p}$ due to cooling at the equilibrium is also shown respectively for the betatron oscillations and for the momentum plane.

For Tevatron I the effect of relativistic cooling is significant but not very large. It is possible to reduce the emittance by a factor of four. This will correspond to twice less protons or correspondingly higher luminosity. Moreover the cooling time of 12 hours should lengthen considerably the luminosity ifetime.

For the case of the Spp̄s the cooling is very effective. In principle the emittance can be lowered by a factor of 200 which would yield a considerable saving on the required number of antiprotons. Nevertheless the beam-beam tune shift is now quite large and it is not clear whether this can be sustained with a damping time of 7 seconds, since this may not be short enough. Obviously there is a draw-back for this significant result: it is required to get to this very small emittance before the cooling becomes that effective. With the initial value of $18 \pi \mathrm{mm}$-mrad the cooling time is a'long period of about two hours, still of some practical interest. Of course one could quickly gain on the results by reducing the initial emittance of both beams either with an improved injector and/or more effective stochastic cooling.

\section{Fast Momentum Cooling}

As shown in Table IV the cooling time on the momentum plane is extremely short for both the Spps and Tevatron $I$. We have explained this as due to the relativistic transformation as one can derive by comparing eq. (9a) with eq. (9b), that is a presence of a factor $\gamma^{2}$ on the momentum cooling rate. Is there any way that one can find some useful application of this fast momentum cooling? For instance if a device could be inserted that would strongly couple longitudinal and transverse oscillations than it may be possible to cool also betatron oscillations at those large rates. 


\section{References}

1. C. Rubbia, Proceed. of Workshop on Producing High-Luminosity High Energy Proton-Antiproton Collisions, March 27-31, 1978, Berkeley, California, p. 98.

2. J. Gareyte, 12 th Intern. Conf. on High Energy Accelerators, Fermilab Illinois, Aug. 1983, p. 17.

3. Design Report Tevatron I Project, Fermilab, Sept. 1984.

4. A.G. Ruggiero "A Proton-Antiproton Superconducting Super Collider". Paper submitted to the proceedings of the 1984 Snowmass workshop.

5. L. Evans and J. Gareyte, US Particle Accelerator Conference, Santa Fe, March $21-23,1983$.

6. L.R. Evans, 12 Intern. Conf. on High Energy Accelerators, Fermilab, Illinois, Aug. 1983, p. 229.

7. B. Richter. Private Communication, SLAC, Stanford, California, 1980.

8. A. Piwinski, IX Intern. Conf. on High Energy Accelerators, Stanford California, May 1974, p. 405.

9. J.D. Bjorken and S.K. Mtingwa, Particle Accelerators, Vol. 13, p. 115, 1983.

10. M. Martini, CERN PS/84-9 (AA) May, 1984.

11. A.G. Ruggiero, Proceed, of Workshop on Producing High-Luminosity High Energy Proton-Antiproton Collisions, March 27-31, 1978, Berkeley, California, p. 166. 
Table I. Proton-Antiproton Colliders

\begin{tabular}{|c|c|c|c|}
\hline & $\begin{array}{c}\operatorname{Sp} \bar{p} S \\
\text { (achieved) }\end{array}$ & Tevatron I & $\mathrm{p} \overline{\mathrm{p}}-\mathrm{SSC}$ \\
\hline Average radius, $\mathrm{km}$ & 1.1 & 1.0 & 104.6 \\
\hline Energy, $\max , \mathrm{TeV}$ & 0.27 & 1.0 & 20. \\
\hline No. of bunches/beam & 3 & 3 & 4428 \\
\hline No. of $\bar{p}^{\prime} \mathrm{s} /$ bunch & $0.15 \times 10^{11}$ & $6 \times 10^{10}$ & $0.77 \times 10^{10}$ \\
\hline No. of $p^{\prime} s / b u n c h$ & $1.4 \times 10^{11}$ & $6 \times 10^{10}$ & $3 \times 10^{10}$ \\
\hline Bunch area, eV-sec & 0.5 & 3.0 & 4.5 \\
\hline $\begin{array}{l}\text { Momentum spread, } \delta \\
\text { Normalized emittan } \mathrm{Be}\end{array}$ & $0.8 \times 10^{-4}$ & $1.2 \times 10^{-4}$ & $0.18 \times 10^{-4}$ \\
\hline$\pi \mathrm{mm}-\mathrm{mrad}$ & 18 & 24 & 24 \\
\hline $\mathrm{n}^{*} \mathrm{H}, \mathrm{V}, \mathrm{m}$ & $1.3 / 0.65 \mathrm{~m}$ & $\begin{array}{l}1.0 \\
0.18\end{array}$ & $\begin{array}{l}1.0 \\
0.0\end{array}$ \\
\hline Luminosity, $\mathrm{cm}^{-2} \mathrm{~s}^{-1}$ & $1.8 \times 10^{29}$ & $1.0 \times 10^{30}$ & $1.0 \times 10^{32}$ \\
\hline $\begin{array}{l}\text { Beam-beam tune-shift } \\
\text { Antiproton Production }\end{array}$ & 0.0045 & 0.0017 & 0.00082 \\
\hline rate, $/ \mathrm{sec}$ & $2.5 \times 10^{-6}$ & $3.5 \times 10^{-7}$ & $8 \times 10^{-8}$ \\
\hline Filling time $(\bar{p})$ & $24 \mathrm{~h}$ & $1.5 \mathrm{~h}$ & $12 \mathrm{~h}$ \\
\hline
\end{tabular}

Table II. Collider Performance vs. Emittance

Normalized

Emittance

$24 \pi \mathrm{mm}-\mathrm{mrad}$,

6

1

0.24

0.06

0.01

\section{Spps Tevatron I p $\bar{p}-S S C$}

$\stackrel{\mathrm{N}}{\mathrm{N}} \mathrm{BB}$

$N / \varepsilon$

0.0045

0.0017

0.00082

1

1

$\begin{array}{ll}0.0034 & 0.00164 \\ 0.5 & 0.5 \\ 2 & 2\end{array}$

$\Delta \nu_{B B}$
$N / \varepsilon$

0.009

2

$\underset{N}{N} \nu_{B B}$

0.022

0.2

5

0.0083

0.2

5

0.004

0.2

5

0.045

0.017

0.1

0.0082

${ }_{N}^{\Delta \nu_{B B}}$

0.1

10

10

$N / \varepsilon$

0.09

20

0.034

0.0164

${ }_{N}^{\Delta v_{B B}}$

20

0.05

$\mathrm{N} / \varepsilon$

0.22

0.02

50
0.085

0.02

50
20
${ }_{\mathrm{N}}^{\Delta \nu_{\mathrm{BB}}}$
$N / E$

0.04

0.02

50 
- Table III Electron Storage Ring Parameters

\begin{tabular}{|c|c|c|c|}
\hline & Spp̄s & Tevatron I & $p \bar{p}-S S C$ \\
\hline$\gamma$ & 270 & 1000 & 20,000 \\
\hline Circumference & $30 \mathrm{~m}$ & $50 \mathrm{~m}$ & $1,000 \mathrm{~m}$ \\
\hline Bending radius* & $1 \mathrm{~m}$ & $2 \mathrm{~m}$ & $40 \mathrm{~m}$ \\
\hline Betatron tune & & 10 & 200 \\
\hline Energy loss/turn & $30 \mathrm{eV}$ & $2.8 \mathrm{KeV}$ & $22 \mathrm{MeV}$ \\
\hline Damping time, $\tau$ & $450 \mathrm{msec}$ & $30 \mathrm{msec}$ & $15 \mathrm{msec}$ \\
\hline rms energy spread, $\delta_{e}$ & $1.2 \times 10^{-4}$ & $3.1 \times 10^{-4}$ & $1.4 \times 10^{-3}$ \\
\hline$B$-emittance, $\bar{\varepsilon} \bar{e}_{e}$ & $3.1 \times 10^{-10} \mathrm{~m}$ & $7.6 \times 10^{-10} \mathrm{~m}$ & $3.8 \times 10^{-11} \mathrm{~m}$ \\
\hline $\begin{array}{l}B e^{*}(H \text { and } V) \\
\text { Interaction length }\end{array}$ & $\begin{array}{l}70 \mathrm{~m} \\
10 \mathrm{~m}\end{array}$ & $\begin{array}{l}70 \mathrm{~m} \\
10 \mathrm{~m}\end{array}$ & $\begin{array}{l}200 \mathrm{~m} \\
200 \mathrm{~m}\end{array}$ \\
\hline
\end{tabular}

(*) With a bending field of about $10 \mathrm{KG}$.

Table IV. Performance with Relativistic Electron Cooling

\begin{tabular}{|c|c|c|c|}
\hline & Sp p S & Tevatron I & $p \bar{p}-S S C$ \\
\hline$\gamma$ & 270 & 1000 & 20,000 \\
\hline$\varepsilon_{n}$ & $6 \times 10^{-11} \mathrm{~m}$ & $9 \times 10^{-20} \mathrm{~m}$ & $1.6 \times 10^{-6} \mathrm{~m}$ \\
\hline$f_{0}^{p}$ & 5 & 1 & $2.4 \times 10^{-5}$ \\
\hline$\varepsilon_{N}^{0}$ & $0.1 \pi \mathrm{mm}-\mathrm{mrad}$ & $5.4 \pi \mathrm{mm}-\mathrm{mrad}$ & - \\
\hline$N^{N}$ & $10^{10}$ & $3 \times 10^{10}$ & - \\
\hline$I_{D}^{P}$ & $0.5 \mathrm{amp}$ & $1.5 \mathrm{amp}$ & - \\
\hline $\mathrm{I}_{\mathrm{N}}^{\mathrm{e}}$ & 50 amp & $150 \mathrm{amp}$ & - \\
\hline $\mathrm{N}_{\mathrm{e}}$ & $10^{12}$ & $3 \times 10^{12}$ & \\
\hline$\tau_{B}$ & $7 \mathrm{sec}$ & 12 hour & - \\
\hline$\tau_{p}^{D}$ & $0.2 \mathrm{msec}$ & $80 \mathrm{msec}$ & - \\
\hline$\Delta \mathrm{P}_{\mathrm{BB}}$ & 0.05 & 0.0034 & - \\
\hline$\overline{\mathrm{p}}$ - & $2-3$ hour & $<1$ hour & - \\
\hline
\end{tabular}




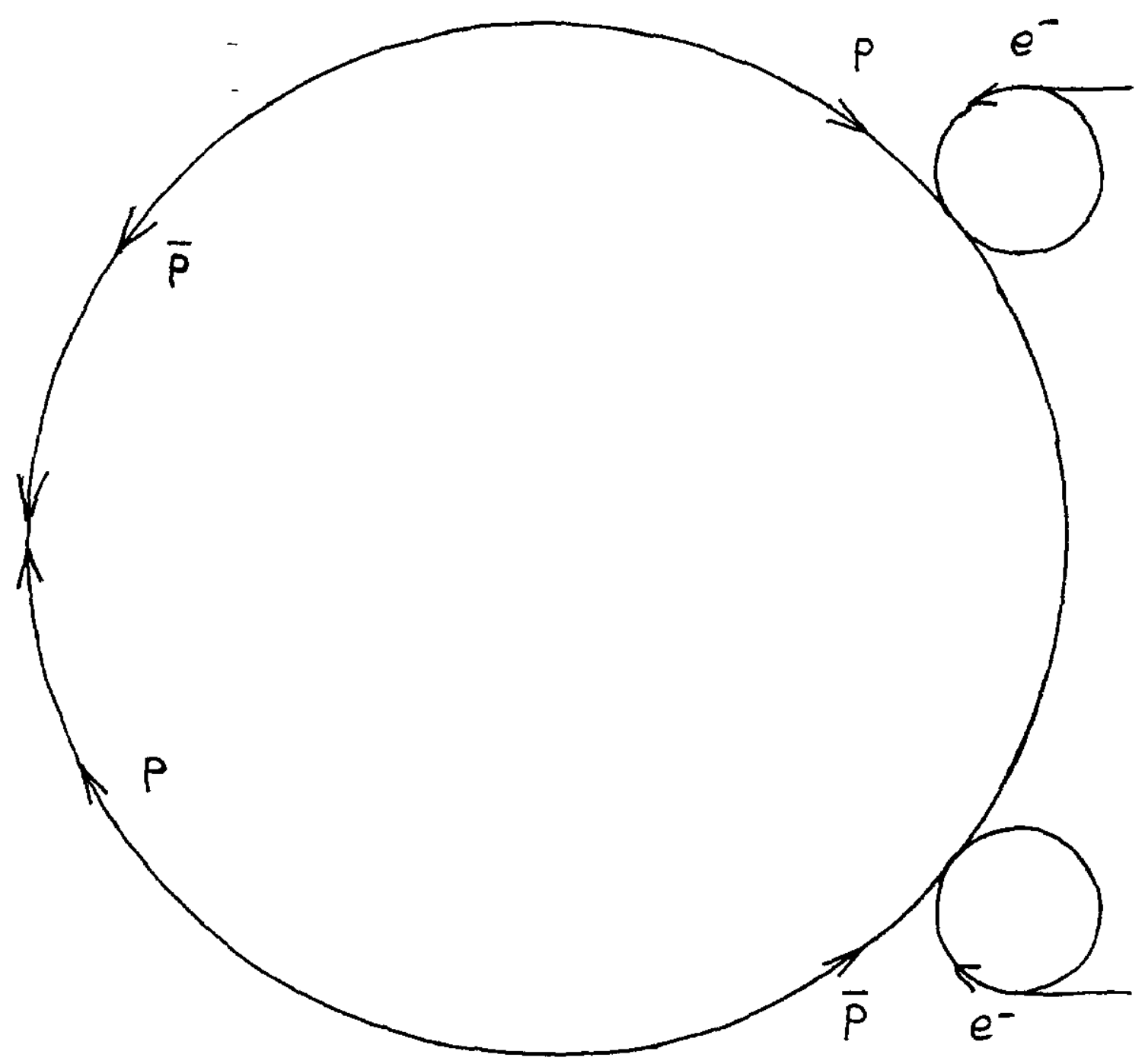
1. $\operatorname{Spp} S$
$E_{p}=270 \mathrm{GeV}$
$E_{e}=135 \mathrm{MeV}$
2. $T e V I$
$1 \mathrm{TeV}$
$500 \mathrm{MeV}$
3. $p \bar{p}-S S C$
$20 \mathrm{TeV}$
$10 \mathrm{GeV}$

Fig. I Layout of a $p \bar{p}$ Collider with $e^{-}$ Storage Rings for Relativistic Electron Cooling 


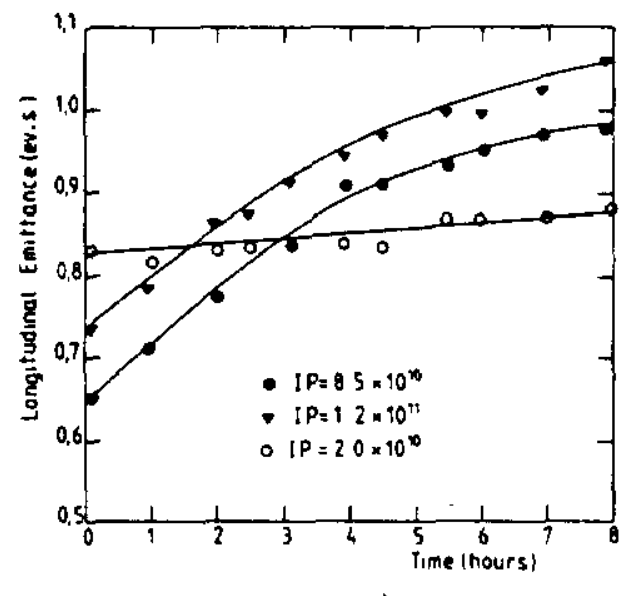

a)

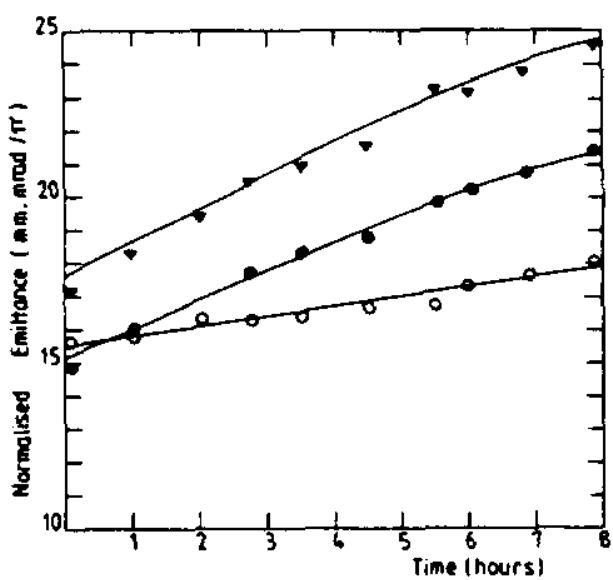

b)

Pis. 2 Longitudinal a) and radiel b) enittance growth of throe proton bunchee witb different intenatien

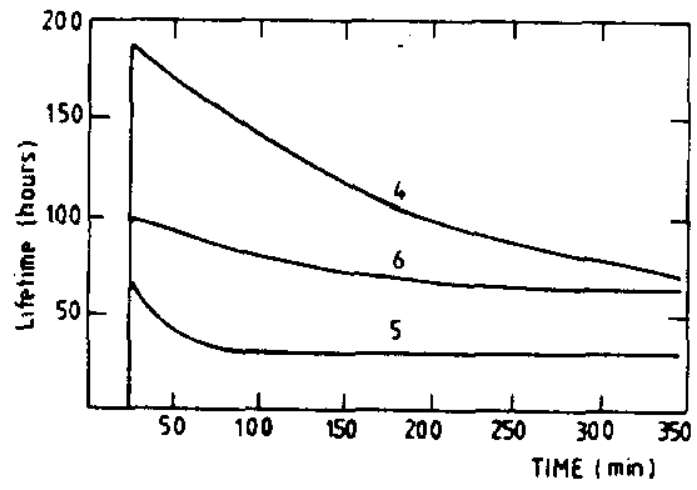

Ris. 3 Lifetime evolution of three proton bunches with dffecent longitudinal omittence and intenelty. 